Sabrina Malvagia, Lorenzo Ferri, Maria Della Bona, Walter Borsini, Calogero Lino Cirami, Egrina Dervishi, Sandro Feriozzi, Serena Gasperini, Serena Motta, Renzo Mignani, Barbara Trezzi, Federico Pieruzzi, Amelia Morrone, Marta Daniotti, Maria Alice Donati and Giancarlo la Marca*

\title{
Multicenter evaluation of use of dried blood spot compared to conventional plasma in measurements of globotriaosylsphingosine (LysoGb3) concentration in 104 Fabry patients
}

https://doi.org/10.1515/cclm-2021-0316

Received March 16, 2021; accepted April 20, 2021;

published online April 30, 2021

\section{Abstract}

Objectives: Fabry disease (FD) is an X-linked lysosomal storage disorder, resulting from a deficiency of the enzyme

*Corresponding author: Giancarlo la Marca, Pharm Sc. Associate Professor, Newborn Screening, Clinical Chemistry and Pharmacology Lab, Meyer Children's University Hospital, Florence, viale Pieraccini 24, Italy; and Department of Experimental and Clinical Biomedical Sciences, University of Florence, Florence, Italy, Phone: +39055 5662988, E-mail: giancarlo.lamarca@meyer.it. https://orcid.org/ 0000-0003-3319-7260

Sabrina Malvagia and Maria Della Bona, Newborn Screening, Clinical Chemistry and Pharmacology Lab, Meyer Children's University Hospital, Florence, Italy

Lorenzo Ferri, Molecular and Cell Biology Laboratory of Neurometabolic Diseases, Neuroscience Department, Meyer Children's Hospital, Florence, Italy

Walter Borsini, Casa di Cura Villa Ulivella e Glicini, Florence, Italy Calogero Lino Cirami and Egrina Dervishi, Nephrology Dialysis Transplant Unit, Careggi Hospital, Florence, Italy

Sandro Feriozzi, Nephrology and Dialysis Unit, Belcolle Hospital, Viterbo, Italy

Serena Gasperini and Serena Motta, Pediatric Rare Diseases Unit, Department of Pediatrics, MBBM Foundation, San Gerardo Hospital, Monza, Italy

Renzo Mignani, Department of Nephrology, Infermi Hospital, Rimini, Italy

Barbara Trezzi, Clinical Nephrology, School of Medicine and Surgery, University of Milano, Milan, Italy

Federico Pieruzzi, Clinical Nephrology, School of Medicine and

Surgery, University of Milano-Bicocca and Nephrology and Dialysis

Unit, ASST-Monza San Gerardo Hospital, Monza, Italy

Amelia Morrone, Molecular and Cell Biology Laboratory of

Neurometabolic Diseases, Neuroscience Department, Meyer

Children's Hospital, Florence, Italy; and Department of Neurofarba, University of Florence, Florence, Italy

Marta Daniotti and Maria Alice Donati, Metabolic Disease Unit, Meyer Children's University Hospital, Florence, Italy $\alpha$-galactosidase A, responsible for breaking down glycolipids such as globotriaosylceramide and its deacylated derivative, globotriaosylsphingosine (LysoGb3). Here, we compare the levels of LysoGb3 in dried blood spots (DBS) and plasma in patients with classic and late-onset phenotypes.

Methods: LysoGb3 measurements were performed in 104 FD patients, 39 males and 65 females. Venous blood was collected. A portion was spotted onto filter paper and another portion separated to obtain plasma. The LysoGb3 concentrations in DBS and plasma were determined by highly sensitive electrospray ionization liquid chromatography tandem mass spectrometry. Agreement between different matrices was assessed using linear regression and Bland Altman analysis.

Results: The method on DBS was validated by evaluating its precision, accuracy, matrix effect, recovery, and stability. The analytical performances were verified by comparison of a total of 104 paired DBS and plasma samples from as many FD patients (representing 46 GLA variants). There was a strong correlation between plasma and the corresponding DBS LysoGb3 concentrations, with few exceptions. Discrepancies were observed in anemic patients with typically low hematocrit levels compared to the normal range.

Conclusions: The method proved to be efficient for the rapid analysis of LysoGb3. DBS provides a convenient, sensitive, and reproducible method for measuring LysoGb3 levels for diagnosis, initial phenotypic assignment, and therapeutic monitoring in patients with FD.

Keywords: dried blood spot; Fabry disease; globotriaosylsphingosine; liquid chromatography-tandem mass spectrometry; LysoGb3.

\section{Introduction}

Fabry disease (FD, OMIM 301500) is caused by an inborn error of glycosphingolipid metabolism, a genetic defect 
resulting in partial or complete deficiency of the lysosomal hydrolase alpha-galactosidase A (alpha-Gal A) [1]. The $\alpha$-galactosidase gene (GLA; OMIM *300644) is located in the Xq22 region. Consequently, FD has an X-linked inheritance so the disease expression is full in males while in heterozygous females is variable depending on the $\mathrm{X}$-chromosome inactivation [2]. Alpha-Gal A deficiency results in an inability to hydrolyze certain glycolipids, primarily globotriaosylceramide (Gb3) which accumulates in the lining of the blood vessels within the kidney, heart, skin, brain and other organs together with other glycoconjugates with $\alpha$-galactosyl terminal moieties, including the deacylated derivative of $\mathrm{Gb} 3$, globotriaosylsphingosine [3]. The progressive accumulation of these lipids in the blood vessels is at the basis of clinical manifestations such as kidney failure, stroke, cardiovascular disease, severe pain and numbness [4].

In males, the classical Fabry phenotype generally arises in the pediatric age with acroparesthesia, hypo- or anhidrosis, angiokeratoma, corneal opacities (typically cornea verticillata) and microalbuminuria. At a later age, progressive kidney disease, hypertrophic cardiomyopathy and cerebrovascular disease (including stroke) can occur. In addition to the classical phenotype, an increasing number of patients with an attenuated late-onset phenotype are diagnosed. These patients have been described in the literature with symptoms affecting one or several organs, mainly the cardiovascular system.

More than a thousand GLA variants associated with Fabry disease have been identified (Human Gene Mutation Database - HGMD Professional, 2020.4 update, https:// my.qiagendigitalinsights.com/bbp/view/hgmd/pro/start. php), most of which occur in single families [5]. FD is a rare pan-ethnic disorder affecting the general population with a wide incidence range, approximately 1:17.000 to 1:117.000 subjects [6].

Some newborn screening programs (NBS) have found an incidence much higher than clinically diagnosed cases $[7,8]$. Nevertheless, genetic variants detected in newborns with low enzymatic activity may never result in clinical phenotype. The misclassification of variants can represent a serious challenge in gene testing results, which may result in inappropriate treatment $[9,10]$.

FD diagnosis remains a challenge due to the complexity of its genetics and the wide spectrum of symptoms which overlap with clinical manifestations of other diseases. A multidisciplinary approach basing on clinical findings, genetic analysis and biochemical investigations is mandatory to confirm FD.

Recently, LysoGb3 was found to be a useful biomarker in confirming FD diagnosis even in symptomatic females with normal or borderline leukocyte enzyme activity due to the effect of X-chromosome inactivation [11, 12].

Specific treatment for FD is based on enzyme replacement therapy (ERT). Chaperone molecules and supportive medications are also available. The main goal of the treatment is to prevent the disease progression to severe organ damage. ERT may successfully decrease the cardiac mass in Fabry hypertrophic cardiomyopathy, reduces Gb3-accumulation in renal endothelia and significantly lower the incidence of renal, cardiovascular and cerebrovascular events, while the effects on the nervous system and renal function are less well established [13-16].

Recent advances in the treatment of FD include gene therapy and the use of Lucerastat, a glucosylceramide synthase inhibitor for reducing of glycosphingolipids. This prevents the accumulation of Gb3 inside the cells [17, 18].

LysoGb3 concentrations are a valid biochemical parameter for determining the time to initiate a pharmacological treatment and for monitoring and evaluating treatment progress $[19,20]$.

Over the last few years, a number of validated methods for measuring LysoGb3 on plasma have been reported, normally requiring a large amount of whole blood (3-5 mL) to produce sufficient sample for the analysis (varying between 50 and $200 \mu \mathrm{L}$ of plasma) [21-26]". In paediatric patients, more specifically in newborns, the available amount of whole blood is generally very limited. Taking larger blood samples is impractical and hampered by ethical issues.

Dried blood spot (DBS) sampling represents an optimized method of sample collection because it is minimally invasive, ensures plasma molecules' stability on filter paper and allows samples to be easily transported and stored $[27,28]$. For these reasons, several researchers have evaluated the use of DBS for measuring LysoGb3 [29-31]. However, the previously published methods lacked technical validation in the comparison between DBS vs. traditional serum/ plasma concentrations or the interesting comparative results, were based on a limited numbers of patients [32-34].

Here, we report results from an observational prospective study where the correlation between DBS and plasma LysoGb3 levels was evaluated in 39 males and 65 females affected with one or other of the FD phenotypes.

\section{Materials and methods}

\section{Study design}

The prospective observational study aimed to enroll at least 100 Fabry patients (50\% males and $50 \%$ females) between the ages of 4 and 75 
years from six clinical centres in Italy and at least 200 age and sex matched controls. The aim of the project was to measure LysoGb3 concentrations in plasma and DBS samples, comparing the sensitivities and specificities of correlated values.

\section{Patients}

The Institutional Ethics Board of Meyer Hospital of Florence approved this study (No. 116/2017). Informed consent was obtained from all patients or their legal guardians prior to their participation.

The authors have complied with the World Medical Association Declaration of Helsinki regarding ethical conduct of research involving human subjects.

The clinical study involved six "FD clinical centres" and 104 patients ( 39 males and 65 females). In all patients the diagnosis of Fabry disease had been previously established using criteria published by Biegstraaten et al. [35].

For each case, two age and sex matched controls were selected (ratio 1:2). The subjects were divided into four age groups: 4-10 years $(n=8) ; 11-17$ years $(n=7) ; 18-49$ years $(n=50)$ and $50-75$ years $(n=39)$. Plasma and DBS samples from 208 healthy donors were collected after anonymization for the assessment of normal range/group. Plasma and DBS samples were stored at $-20^{\circ} \mathrm{C}$ until they were analysed.

\section{Chemical and reagents}

Water, acetonitrile and methanol ( $₫ 99.9 \%$, MS grade) were supplied by Biosolve (Chebios, Rome-Italy). Formic acid and acetone (ACS reagent grade) were from Sigma-Aldrich (Milan, Italy). LysoGlobotriaosylsphinosine (lyso-Gb3) was purchased from Matreya LLC (State College, PA, USA).

\section{Sample preparation}

A $3.2 \mathrm{~mm}$ diameter disk from DBS ( $\sim 3 \mu \mathrm{L}$ blood) was punched directly into a 96-well plate and extracted with $150 \mu \mathrm{L}$ of methanol. The well plate was closed with a polypropylene plate cover and then enveloped with aluminium foil; extraction was performed in an orbital shaker for $20 \mathrm{~min}$ at room temperature $\left(+24^{\circ} \mathrm{C} \pm 1{ }^{\circ} \mathrm{C}\right)$ then the well plate was centrifuged for $10 \mathrm{~min}$ at $3,000 \mathrm{rpm}$. The extract supernatant was transferred to a clean 96-well plate, sealing with plastic film and then injected into the LC/MS/MS system.

For plasma samples, $50 \mu \mathrm{L}$ were diluted with $200 \mu \mathrm{L}$ of acetone/ methanol/water $(45 / 45 / 10, \mathrm{v} / \mathrm{v} / \mathrm{v})$ in a $1.5 \mathrm{~mL}$ Eppendorf tube (Eppendorf, Milano, Italy), vortexed for $20 \mathrm{~s}$ and then centrifuged at $16,100 \mathrm{~g}$ to precipitate proteins. The extract was transferred to a $2 \mathrm{~mL}$ glass vial (Agilent Technologies, Santa Clara, CA, USA) and further evaporated under nitrogen flow. The dried sample was reconstituted in $150 \mu \mathrm{L}$ of acetonitrile/water $(70 / 30, \mathrm{v} / \mathrm{v})$ with $0.1 \%$ formic acid and immediately analyzed.

\section{Validation procedure}

The analytical method was validated for selectivity, linearity, lower limit of quantification (LLOQ), accuracy, precision, matrix effect, and stability in accordance with ICH harmonised guidelines [36].
Calibration curve and quality controls (QC): A standard stock solution of LysoGb3 was prepared at a concentration of $1.3 \mathrm{mmol} / \mathrm{L}$ chloroform/ methanol $(75 / 25, \mathrm{v} / \mathrm{v})$ and stored at $-20{ }^{\circ} \mathrm{C}$. Two sets of the same standard stock solution were prepared for standard curves and for quality control (QC) samples, respectively. Working standard solutions of different concentrations were prepared by diluting stock solutions with methanol.

A standard curve of LysoGb3 was constructed by spiking human plasma from healthy controls with the standard working solutions (dilution 1:100) to obtain eight point levels ranging from 0.50 to $100 \mathrm{nmol} / \mathrm{L}(0.5,1,2.5,5,10,25,50,100 \mathrm{nmol} / \mathrm{L})$. Another calibration curve on DBS, covering a concentration range from 1 to $100 \mathrm{nmol} / \mathrm{L}$, was prepared by spiking different LysoGb3 standard solutions into whole blood from a healthy volunteer blood donor with known hematocrit (Hct). To prevent changes in blood composition, the spiked blood preparation was performed as follows: for each level concentration, a $10 \mu \mathrm{L}$ aliquot of a 100 times concentrated working standard solution was transferred into a clean glass vial and dried under nitrogen gas flow for rapidly removing organic solvents. Then, $1 \mathrm{~mL}$ of whole blood was added to each vial, vortex and spotted on filter paper (Whatman, $903^{\circledR}$, Gmbh, Dassel Germany). Similarly, QC samples on DBS were prepared at concentrations of 1, 2, 10 and $75 \mathrm{nmol} / \mathrm{L}$, representing values close to LOQ, medium and high levels. All DBS samples were stored in zip lock plastic bags at $-20^{\circ} \mathrm{C}$ until analysis.

Limit of detection and limit of quantitation: The limit of detection (LOD) and the limit of quantitation (LOQ) were determined by preparing for whole blood and plasma a specific calibration curve in blood and plasma in the range $0-2 \mathrm{nmol} / \mathrm{L}$; the residual standard deviation of the regression line and its slope were used to calculate LOD and LOQ.

Accuracy and precision: The accuracy and precision of the method were carried out by analysing the DBS and the plasma QC samples in 10 replicates for intraday test and in duplicate for 10 different days for inter-day test. The accuracy was determined by comparing the measured and known values.

Recovery and matrix effect: The efficiency of the extraction procedure was also determined by analysis of DBS and plasma at three different concentration levels, 2,10 and $75 \mathrm{nmol} / \mathrm{L}$ in triplicate over three days. The analyte response obtained from extracted spiked samples was compared to a spiked blank matrix sample and the recovery was calculated.

The matrix effect was calculated by comparing the signal obtained by LysoGb3 at 2, 10 and $75 \mathrm{nmol} / \mathrm{L}$ concentrations, injected directly in the mobile phase and the signal obtained from the same amount of compound added to an extracted LysoGb3 free matrix.

Spot homogeneity: Spot homogeneity was also verified by punching at different locations, including left, right, centre, top and bottom edge of the QC samples (low and high levels). The QC spots were obtained by depositing $25 \mu \mathrm{L}$ of whole blood (Hct 0.55 ) on $903^{\circledR}$ filter paper. Three replicates were tested at each $\mathrm{QC}$ level.

Stability and storage: The short-term stability on DBS was evaluated up to four weeks under the following storage conditions: $-20^{\circ} \mathrm{C},+4^{\circ} \mathrm{C}$, room temperature and $+37^{\circ} \mathrm{C}$ to ensure that patient samples are stable during shipping and storage prior to analysis. The stability of DBS 
samples during storage was determined weekly by analysing QC samples (four level concentrations; $\mathrm{n}=3$ replicates).

\section{LC-MS/MS analysis}

The data were obtained using an API 5,500 QTRAP mass spectrometer (AB SCIEX, Toronto, Canada) equipped with Turbo Ion Spray source and operating in MRM positive ion mode. The Ion spray voltage was set to $5,500 \mathrm{~V}$, gas 1 and gas 2 were both set at 40 (arbitrary unit) and the temperature was $500{ }^{\circ} \mathrm{C}$.

The transitions were optimized by infusing a standard solution $(1.3 \mu \mathrm{mol} / \mathrm{L})$ and the following MS/MS conditions were monitored: the transition $\mathrm{m} / z 786.6>282.3$ was used as quantifier, with $45 \mathrm{~V}$ as collision energy voltage, the transition $786.6>264.3$ was used as qualifier, $40 \mathrm{~V}$ as collision energy voltage (Figure 1).

Chromatographic separation was achieved using an Agilent 1290 Infinity UHPLC capillary system (Agilent Technologies, Waldbronn, Germany), operating in gradient mode, coupled with a thermostated autosampler and fully controlled by Analyst Software (Version 1.5.2).

The chromatographic run was performed by using a Kinetex C8 solid core column, $2.6 \mu \mathrm{m}, 50 \times 2.1 \mathrm{~mm}$ (Phenomenex, Torrance, CA, USA) at a flow rate of $400 \mu \mathrm{L} / \mathrm{min}$; the eluate was flowed directly into the ESI source without splitting. The mobile phase was composed of water (phase A) and acetonitrile (phase B), both with $0.1 \%$ formic acid added.

Separation was achieved using a step gradient starting with $20 \%$ (phase B) maintained for 0.5 min after which the mobile phase was set at $50 \%$ (phase B) to be reached in $0.75 \mathrm{~min}$. After that, the mobile phase reached $75 \%$ (phase B) in 1.25 min followed by a step to $95 \%$ (phase B) in $0.5 \mathrm{~min}$. The mobile phase was returned to the starting conditions within $0.1 \mathrm{~min}$ and re-equilibrated for $1.4 \mathrm{~min}$. The total run time was $4.5 \mathrm{~min}$ and the LysoGb3 retention time was $1.95 \mathrm{~min}$. During the analysis, the column was maintained in the UHPLC oven at $45^{\circ} \mathrm{C}$. The injection volume was $2 \mu \mathrm{L}$, and the autosampler temperature was maintained at $4{ }^{\circ} \mathrm{C}$ throughout the analyses.

The data obtained were elaborated using Analyst software 1.5.2 and the calibration curves were set-up using a linear least square nonweighted regression.

\section{Results and discussion}

Differences on LysoGb3 quantification related to DBS punch location that we observed were lower than $15 \% \mathrm{CV}$, therefore negligible. In order to identify the best extraction solution for LysoGb3, different solvents and mixtures were tested. The best extraction efficiency for DBS samples was obtained by using methanol whereas a mixture of acetone/ methanol/water (45/45/10, v/v/v) was chosen for plasma samples. In addition, different solvent volumes, extraction times and temperatures were investigated to maximize extraction yields. The amount of solvent required for complete extraction of LysoGb3 from DBS was verified by performing a second extraction on the exhausted DBS. Using $150 \mu \mathrm{L}$ of solvent, the amount of LysoGb3 obtained after a second extraction represented less than $4 \%$ of the previous one. Extraction efficiency was also tested at

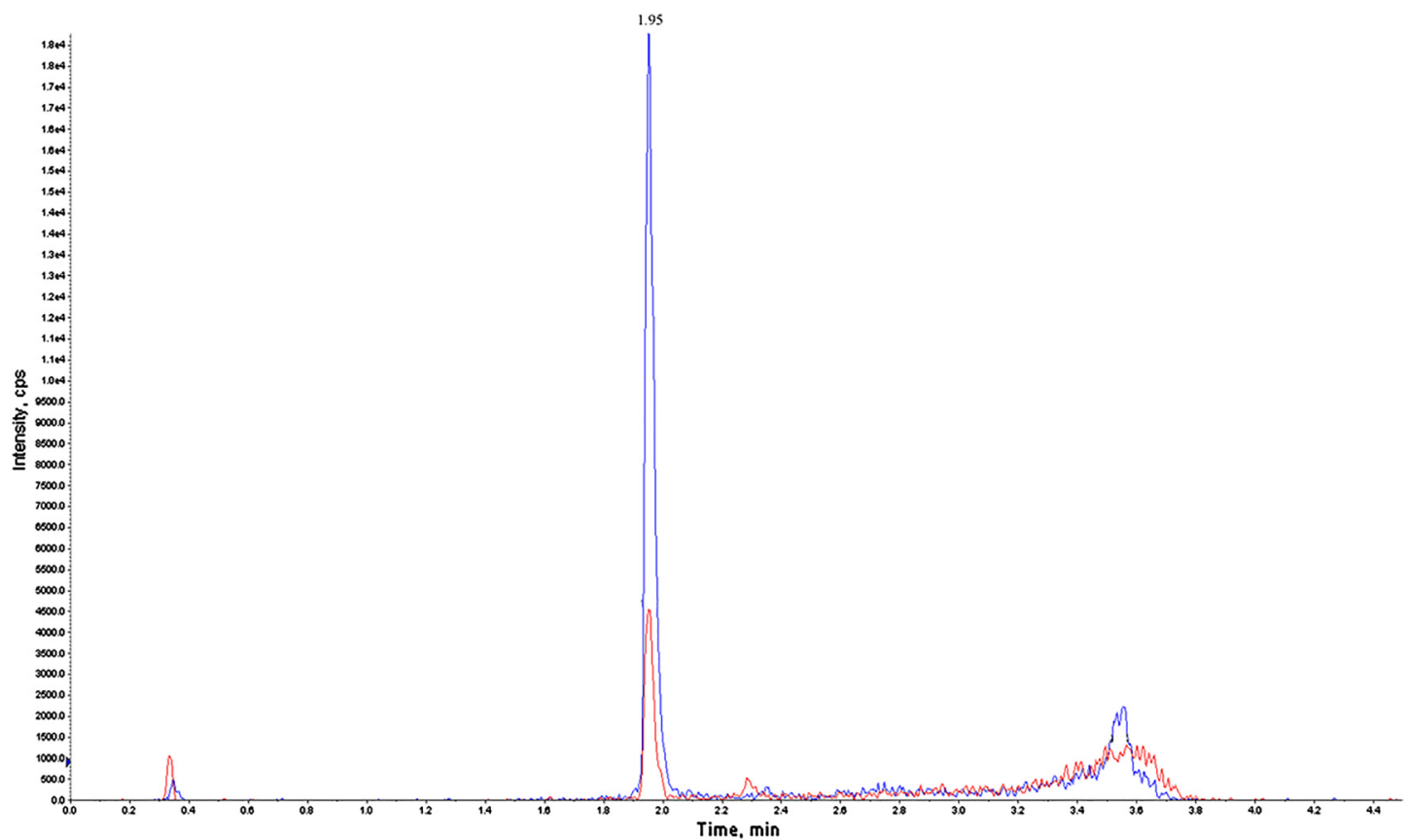

Figure 1: Representative extracted ion chromatogram of a DBS sample fortified to $50 \mathrm{nmol} / \mathrm{L}$ with LysoGb3. For quantitation, two MRM transitions were chosen and scanned during the LC gradient. The blue peak is the resulting ion-pair transition $\mathrm{m} / \mathrm{z} 786.6>282.3$ for the quantitative experiment (quantifier). The read peak is the resulting ion-pair transition $\mathrm{m} / \mathrm{z} 786.6>264.3$ to definitively identify the presence of LysoGb3 (qualifier). 
different temperatures, extraction times and static or notstatic conditions. The best conditions were $20 \mathrm{~min}$ at room temperature extracting in an orbital shaker. The percentage recovery ranged from 98.7 to $106 \%$ over three days for all levels.

Possible effects of paper were also investigated by comparing a standard solution injection and the corresponding concentration spotted on paper without matrix and then extracted: no difference was detected. No matrix effect was observed for any QC level.

Chromatographic conditions were tested using acetonitrile and methanol as organic mobile phases: acetonitrile was selected because of a negligible memory effect compared to methanol where the memory effect was significantly high.

The aim of this work was to develop and validate a simple and rapid method to quantify LysoGb3 in DBS

Table 1: Intra-day and inter-day accuracy and precision of LysoGb3 on DBS.

\begin{tabular}{lrrrr}
\hline $\begin{array}{l}\text { Expected concentration, } \\
\text { nmol/L }\end{array}$ & $\begin{array}{r}\text { Mean, } \\
\mathbf{n g} / \mathbf{m L}\end{array}$ & $\begin{array}{r}\text { Precision, } \\
\mathbf{C V} \%\end{array}$ & $\begin{array}{r}\text { RSD, } \\
\%\end{array}$ & $\begin{array}{r}\text { Accuracy, } \\
\%\end{array}$ \\
\hline Intra-day (n=10, 1 day) & & & & \\
1 & 1.1 & 5.4 & 0.1 & 105.6 \\
2 & 2.0 & 5.8 & 0.1 & 101.3 \\
10 & 10.1 & 3.4 & 0.3 & 100.9 \\
75 & 75.7 & 4.1 & 3.1 & 101.0 \\
\hline Inter-day (n=2, 10 days) & & & & \\
1 & 1.1 & 4.9 & 0.1 & 106.0 \\
2 & 2.0 & 3.4 & 0.1 & 102.0 \\
10 & 10.0 & 2.0 & 0.2 & 100.1 \\
75 & 76.1 & 3.3 & 2.6 & 101.1 \\
\hline
\end{tabular}

samples. Quantification was performed using the external calibration method. A good linearity $\left(r^{2}=0.999\right)$ was obtained in the concentration range from 1.0 to $100 \mathrm{nmol} / \mathrm{L}$. A correlation coefficient $>0.995$ is generally considered as evidence that the data fits the regression line.

For determining the LOD and LOQ of the method, a calibration curve with concentrations close to the limit of quantitation was prepared. LOD and LOQ were calculated by using the following equation: $\mathrm{LOD}=3.3 \sigma / \mathrm{S}$ and $\mathrm{LOQ}=10 \sigma / \mathrm{S}$, where $\sigma=$ residual standard deviation and $\mathrm{S}=$ the slope of the regression line. LOD and LOQ were found to be $0.30 \mathrm{nmol} / \mathrm{L}$ and $0.90 \mathrm{nmol} / \mathrm{L}$ in DBS respectively and $0.14 \mathrm{nmol} / \mathrm{L}$ and $0.46 \mathrm{nmol} / \mathrm{L}$ in plasma.

The variations in intra-day and inter-day LysoGb3 determinations on DBS are reported in Table 1. All method validation parameters tested were fully satisfactory.

Good stability of LysoGb3 on DBS (100\%) was observed up to 30 days at $4^{\circ} \mathrm{C}$ or $-20^{\circ} \mathrm{C}$. Storage at $37^{\circ} \mathrm{C}$ and at RT for up 30 days significantly reduced high LysoGb3 levels by 28 and $22 \%$, respectively although surprisingly experimental LysoGb3 concentrations lower than $10 \mathrm{nmol} / \mathrm{L}$ were stable at all tested temperatures. The experimental overall good stability of LysoGb3 makes DBS a preferred method for storage and transportation (Table 2) although a fridge or freezer should be used for long term storage

\section{Correlation DBS vs. plasma}

One hundred and four patients (39 males and 65 females) collected by six FD clinical centres were enrolled (Table 3). 46 GLA gene variants resulted to be included in this work, of them 40 variants were previously described as

Table 2: Effect of short term storage temperature on DBS LysoGb3.

\begin{tabular}{|c|c|c|c|c|c|}
\hline Storage temperature & Expected concentration, $\mathrm{nmol} / \mathrm{L}$ & Mean, $n=3$ (30 days) & RSD & CV, \% & Accuracy, \% \\
\hline & 1 & 1.02 & 0.07 & 6.5 & 102 \\
\hline & 2 & 1.93 & 0.07 & 3.5 & 96 \\
\hline \multirow[t]{4}{*}{$-20^{\circ} \mathrm{C}$} & 10 & 10.31 & 0.51 & 5.0 & 103 \\
\hline & 75 & 81.23 & 5.28 & 6.5 & 108 \\
\hline & 1 & 1.02 & 0.03 & 2.7 & 102 \\
\hline & 2 & 1.97 & 0.10 & 4.8 & 102 \\
\hline \multirow[t]{4}{*}{$+4^{\circ} \mathrm{C}$} & 10 & 10.03 & 0.09 & 0.9 & 100 \\
\hline & 75 & 71.37 & 3.20 & 4.5 & 95 \\
\hline & 1 & 0.94 & 0.14 & 15.4 & 94 \\
\hline & 2 & 2.03 & 0.17 & 8.1 & 102 \\
\hline \multirow[t]{4}{*}{ RT } & 10 & 9.04 & 0.19 & 2.1 & 90 \\
\hline & 75 & 54.02 & 4.29 & 7.9 & 72 \\
\hline & 1 & 1.07 & 0.14 & 12.6 & 107 \\
\hline & 2 & 2.04 & 0.20 & 9.8 & 102 \\
\hline \multirow[t]{2}{*}{$+37^{\circ} \mathrm{C}$} & 10 & 8.52 & 0.19 & 2.2 & 85 \\
\hline & 75 & 58.2 & 3.32 & 5.7 & 78 \\
\hline
\end{tabular}


Table 3: GLA gene variants of the $104 \mathrm{FD}$ patients included in the study.

\begin{tabular}{|c|c|c|c|c|c|c|c|}
\hline & Plasma, nmol/L & DBS, nmol/L & Age, years & Sex & GLA variant & Protein change & Phenotype \\
\hline \multirow[t]{8}{*}{ Group I (4-10 years) } & 52.1 & 52.1 & 6 & M & c. $1117 \mathrm{G}>\mathrm{A}$ & p.Gly373Ser & Classical \\
\hline & 46.6 & 42.4 & 6 & M & c. $1117 \mathrm{G}>\mathrm{A}$ & p.Gly373Ser & Classical \\
\hline & 4.3 & 4.3 & 7 & M & c. $644 A>G$ & p.Asn215Ser & Late-onset \\
\hline & 3.7 & 4.6 & 5 & M & c. $644 A>G$ & p.Asn215Ser & Late-onset \\
\hline & 13.9 & 17.0 & 5 & M & c. $1079 \mathrm{G}>\mathrm{A}$ & p.Gly360Asp & Classical \\
\hline & 2.3 & 2.2 & 9 & $\mathrm{~F}$ & c. $1079 \mathrm{G}>\mathrm{A}$ & p.Gly360Asp & Classical \\
\hline & 4.9 & 4.2 & 5 & $\mathrm{~F}$ & c. $233 C>G$ & p.Ser78* & Classical \\
\hline & 4.2 & 4.7 & 6 & $\mathrm{~F}$ & c. $658 \mathrm{C}>\mathrm{T}$ & p.Arg220* & Classical \\
\hline \multirow[t]{7}{*}{ Group II (11-17 years) } & 115.0 & 102.4 & 11 & M & c. $902 \mathrm{G}>\mathrm{A}$ & p.Arg301Gln & Classical \\
\hline & 267.0 & 261.0 & 11 & M & C. $187 \mathrm{~T}>\mathrm{C}$ & p.Cys63Arg & Classical \\
\hline & 8.4 & 7.1 & 14 & $\mathrm{~F}$ & c. $187 \mathrm{~T}>\mathrm{C}$ & p.Cys63Arg & Classical \\
\hline & 13.3 & 12.4 & 12 & $\mathrm{~F}$ & c.810_812delGinsTCTCA & p.Gly271Leufs*12 & Classical $^{\mathrm{a}}$ \\
\hline & 5.2 & 5.9 & 16 & $\mathrm{~F}$ & c. $901 \mathrm{C}>\mathrm{T}$ & p.Arg301* & Classical \\
\hline & 2.5 & 2.6 & 14 & $\mathrm{~F}$ & c. $272 \mathrm{~T}>\mathrm{C}$ & p.lle91Thr & Late-onset \\
\hline & 5.6 & 5.4 & 11 & $\mathrm{~F}$ & c.635_639+8del & p.Gln212Profs*18 & Classical $^{\mathrm{a}}$ \\
\hline \multirow[t]{40}{*}{ Group III (18-49 years) } & 4.6 & 3.2 & 30 & M & c. $902 \mathrm{G}>\mathrm{A}$ & p.Arg301Gln & Classical \\
\hline & 4.0 & 3.1 & 30 & M & c. $902 \mathrm{G}>\mathrm{A}$ & p.Arg301Gln & Classical \\
\hline & 1.8 & 1.9 & 30 & M & c. $902 \mathrm{G}>\mathrm{A}$ & p.Arg301Gln & Classical \\
\hline & 8.1 & 8.1 & 45 & M & c. $902 \mathrm{G}>\mathrm{A}$ & p.Arg301Gln & Classical \\
\hline & 36.9 & 32.4 & 23 & M & c.1073dup & p.Ile359Aspfs`16 & Classical \\
\hline & 54.8 & 42.9 & 21 & M & c.1073dup & p.lle359Aspfs*16 & Classical \\
\hline & 34.7 & 19.3 & 42 & $M$ & c. $680 \mathrm{G}>\mathrm{C}$ & p.Arg227Pro & Classical \\
\hline & 24.7 & 13.7 & 36 & $M$ & c. $37 \mathrm{G}>\mathrm{C}$ & p.Ala13Pro & Late-onset $^{\mathrm{a}}$ \\
\hline & 44.4 & 23.3 & 38 & $M$ & c.1091_1092delCT & p.Ser364Leufs $* 10$ & Classical \\
\hline & 57.0 & 51.0 & 45 & $M$ & c. $658 \mathrm{C}>\mathrm{T}$ & p.Arg220* & Classical \\
\hline & 46.0 & 51.0 & 30 & M & c. $658 \mathrm{C}>\mathrm{T}$ & p.Arg220* & Classical \\
\hline & 98.0 & 102.4 & 25 & M & $c .4 C>T$ & p.Gln $2^{*}$ & Classical \\
\hline & 43.7 & 38.6 & 29 & M & c.124_125delAT & p.Met42Glyfs ${ }^{\star} 13$ & Classical \\
\hline & 74.5 & 79.9 & 37 & $M$ & c. $1024 C>T$ & p.Arg342* & Classical \\
\hline & 25.3 & 20.7 & 33 & $M$ & c. $515 \mathrm{G}>\mathrm{A}$ & p.Cys172Tyr & Classical \\
\hline & 42.2 & 30.0 & 40 & M & c. $168 C>A$ & p.Cys56* & Classical \\
\hline & 44.3 & 47.3 & 43 & $M$ & c.428dupC & p.Gly144Argfs*12 & Classical \\
\hline & 37.0 & 34.2 & 43 & M & c.618_619delTT & p.Met 208 Valfs $^{\star} 23$ & Classical \\
\hline & 3.4 & 4.3 & 32 & M & c. $644 \mathrm{~A}>\mathrm{G}$ & p.Asn215Ser & Late-onset \\
\hline & 27.5 & 25.0 & 27 & M & c. $388 \mathrm{~A}>\mathrm{G}$ & p.Lys130Glu & Late-onset \\
\hline & 4.5 & 9.3 & 36 & $M$ & c. $1079 \mathrm{G}>\mathrm{A}$ & p.Gly360Asp & Classical \\
\hline & 4.0 & 3.6 & 49 & $\mathrm{~F}$ & c.1073dup & p.Ile359Aspfs*16 & Classical \\
\hline & 0.6 & $<0.9$ & 35 & $\mathrm{~F}$ & c. $335 \mathrm{G}>\mathrm{A}$ & p.Arg112His & Late-onset \\
\hline & 10.6 & 10.1 & 48 & $\mathrm{~F}$ & $c .467 C>T$ & p.Ala156Val & Classical \\
\hline & 5.5 & 5.3 & 42 & $\mathrm{~F}$ & c. $1061 \mathrm{~T}>\mathrm{A}$ & p.Ile354Lys & Classical \\
\hline & 1.9 & 2.0 & 34 & $\mathrm{~F}$ & c. $1061 \mathrm{~T}>\mathrm{A}$ & p.Ile354Lys & Classical \\
\hline & 8.5 & 6.8 & 34 & $\mathrm{~F}$ & c. $1061 \mathrm{~T}>\mathrm{A}$ & p.lle354Lys & Classical \\
\hline & 3.5 & 2.3 & 27 & $\mathrm{~F}$ & c.1073dup & p.Ile359Aspfs`16 & Classical \\
\hline & 27.8 & 20.1 & 44 & $\mathrm{~F}$ & c. $902 \mathrm{G}>\mathrm{C}$ & p.Arg301Pro & Classical \\
\hline & 9.2 & 6.8 & 49 & $\mathrm{~F}$ & c. $902 \mathrm{G}>\mathrm{C}$ & p.Arg301Pro & Classical \\
\hline & 4.3 & 3.6 & 33 & $\mathrm{~F}$ & c.1091_1092delCT & p.Ser364Leufs*10 & Classical \\
\hline & 8.3 & 5.3 & 39 & $\mathrm{~F}$ & c.548-3_553del & p.Gly183Alafs*17 & Classical \\
\hline & 13.1 & 13.2 & 21 & $\mathrm{~F}$ & c.994dupA & p.Arg332Lysfs`7 & Classical \\
\hline & 3.7 & 5.0 & 40 & $\mathrm{~F}$ & c. $1079 \mathrm{G}>\mathrm{A}$ & p.Gly360Asp & Classical \\
\hline & 11.5 & 14.5 & 31 & $\mathrm{~F}$ & c. $658 \mathrm{C}>\mathrm{T}$ & p.Arg220* & Classical \\
\hline & 8.2 & 9.0 & 43 & $\mathrm{~F}$ & c. $901 \mathrm{C}>\mathrm{T}$ & p.Arg301* & Classical \\
\hline & 4.1 & 4.0 & 48 & $\mathrm{~F}$ & c.950T>C & p.lle317Thr & Classical \\
\hline & 5.2 & 6.4 & 37 & $\mathrm{~F}$ & $c .4 C>T$ & p.Gln $2^{*}$ & Classical \\
\hline & 8.2 & 7.8 & 30 & $\mathrm{~F}$ & c. $704 C>G$ & p.Ser235Cys & Classical \\
\hline & 6.4 & 5.8 & 49 & $\mathrm{~F}$ & c. $1146 C>A$ & p.Cys382* & Classical \\
\hline
\end{tabular}


Table 3: (continued)

\begin{tabular}{|c|c|c|c|c|c|c|c|}
\hline & Plasma, nmol/L & DBS, nmol/L & Age, years & Sex & GLA variant & Protein change & Phenotype \\
\hline & 7.0 & 5.3 & 36 & $\mathrm{~F}$ & c.124_125delAT & p.Met42Glyfs*13 & Classical \\
\hline & 6.6 & 5.7 & 43 & $\mathrm{~F}$ & c.635_639+8del & p.Gln $212 \operatorname{Profs}^{\star}{ }^{\star} 18$ & Classical $^{\mathrm{a}}$ \\
\hline & 9.0 & 7.2 & 38 & $\mathrm{~F}$ & c.73delG & p.Asp25Thrfs*96 & Classical \\
\hline & 4.9 & 4.3 & 29 & $\mathrm{~F}$ & c. $1000-1 \mathrm{G}>\mathrm{A}$ & p.? & Classical \\
\hline & 9.5 & 7.4 & 18 & $\mathrm{~F}$ & c. $257 A>G$ & p.Tyr86Cys & Classical \\
\hline & 0.8 & 1.9 & 31 & $\mathrm{~F}$ & c. $644 A>G$ & p.Asn215Ser & Late-onset \\
\hline & $<0.5$ & $<0.9$ & 28 & $\mathrm{~F}$ & c. $1088 \mathrm{G}>\mathrm{A}$ & p.Arg $363 \mathrm{His}$ & Late-onset \\
\hline & 0.8 & 1.1 & 37 & $\mathrm{~F}$ & c. $272 T>C$ & p.lle91Thr & Late-onset \\
\hline & 0.8 & 1.2 & 45 & $\mathrm{~F}$ & c. $644 A>G$ & p.Asn215Ser & Late-onset \\
\hline & 3.8 & 3.8 & 39 & $\mathrm{~F}$ & c. $1079 \mathrm{G}>\mathrm{A}$ & p.Gly360Asp & Classical \\
\hline \multirow[t]{39}{*}{ Group IV (50-77 years) } & 30.3 & 28.2 & 52 & $M$ & c. $1061 \mathrm{~T}>\mathrm{A}$ & p.lle354Lys & Classical \\
\hline & 88.6 & 45.9 & 50 & $M$ & c. $902 \mathrm{G}>\mathrm{C}$ & p.Arg301Pro & Classical \\
\hline & 22.6 & 15.4 & 58 & $M$ & c. $896 \mathrm{~A}>\mathrm{G}$ & p.Asp299Gly & Classical \\
\hline & 23.7 & 17.3 & 55 & $M$ & c. $257 A>G$ & p.Tyr86Cys & Classical \\
\hline & 16.4 & 14.6 & 56 & $M$ & c.781G >T & p.Gly261Cys & Classical $^{\mathrm{a}}$ \\
\hline & 21.1 & 12.7 & 62 & $M$ & c. $334 C>T$ & p.Arg112Cys & Classical \\
\hline & 2.3 & 3.2 & 62 & $M$ & c. $644 A>G$ & p.Asn215Ser & Late-onset \\
\hline & 5.5 & 4.6 & 68 & $M$ & c. $644 A>G$ & p.Asn215Ser & Late-onset \\
\hline & 7.8 & 7.5 & 58 & $M$ & c. $644 A>G$ & p.Asn215Ser & Late-onset \\
\hline & 3.8 & 3.8 & 77 & M & c. $644 A>G$ & p.Asn215Ser & Late-onset \\
\hline & 10.4 & 11.1 & 64 & $M$ & c. $1079 \mathrm{G}>\mathrm{A}$ & p.Gly360Asp & Classical \\
\hline & 4.1 & 3.6 & 69 & $\mathrm{~F}$ & c. $1061 \mathrm{~T}>\mathrm{A}$ & p.lle354Lys & Classical \\
\hline & 2.6 & 3.3 & 80 & $\mathrm{~F}$ & c. $1061 \mathrm{~T}>\mathrm{A}$ & p.lle354Lys & Classical \\
\hline & 5.4 & 4.8 & 59 & $\mathrm{~F}$ & c. $1 A>G$ & p.Met1? & Classical \\
\hline & 4.2 & 3.7 & 66 & $\mathrm{~F}$ & c. $902 \mathrm{G}>\mathrm{C}$ & p.Arg301Pro & Classical \\
\hline & 7.3 & 6.8 & 50 & $\mathrm{~F}$ & c. $1244 \mathrm{~T}>\mathrm{G}$ & p.Leu415Arg & Classical \\
\hline & 6.9 & 6.9 & 60 & $\mathrm{~F}$ & c. $896 A>G$ & p.Asp299Gly & Classical \\
\hline & 8.9 & 5.7 & 73 & $\mathrm{~F}$ & c. $334 \mathrm{C}>\mathrm{T}$ & p.Arg112Cys & Classical \\
\hline & 6.8 & 4.5 & 73 & $\mathrm{~F}$ & c. $902 \mathrm{G}>\mathrm{C}$ & p.Arg301Pro & Classical \\
\hline & 10.8 & 6.2 & 71 & $\mathrm{~F}$ & c.1091_1092delCT & p.Ser364Leufs*10 & Classical \\
\hline & 5.4 & 3.2 & 71 & $\mathrm{~F}$ & c. $728 \mathrm{~T}>\mathrm{C}$ & p.Leu243Ser & Not determined ${ }^{a}$ \\
\hline & 8.0 & 8.2 & 71 & $\mathrm{~F}$ & c. $658 \mathrm{C}>\mathrm{T}$ & p.Arg220* & Classical \\
\hline & 2.4 & 2.2 & 56 & $\mathrm{~F}$ & c. $708 \mathrm{G}>\mathrm{C}$ & p.Trp236Cys & Classical \\
\hline & 10.5 & 10.0 & 50 & $\mathrm{~F}$ & c. $824 \mathrm{~T}>\mathrm{A}$ & p.Leu275His & Classical \\
\hline & 12.1 & 13.5 & 57 & $\mathrm{~F}$ & $c .4 C>T$ & p.Gln $2^{*}$ & Classical \\
\hline & 7.0 & 6.0 & 62 & $\mathrm{~F}$ & c. $233 C>G$ & p.Ser78* & Classical \\
\hline & 2.0 & 1.9 & 63 & $\mathrm{~F}$ & c. $515 \mathrm{G}>\mathrm{A}$ & p.Cys172Tyr & Classical \\
\hline & 3.7 & 4.2 & 56 & $\mathrm{~F}$ & c. $658 \mathrm{C}>\mathrm{T}$ & p.Arg $220^{*}$ & Classical \\
\hline & 6.7 & 6.4 & 68 & $\mathrm{~F}$ & c.428dupC & p.Gly144Argfs*12 & Classical \\
\hline & 5.2 & 5.6 & 53 & $\mathrm{~F}$ & c.781G>T & p.Gly261Cys & Classical $^{\mathrm{a}}$ \\
\hline & 3.7 & 2.7 & 55 & $\mathrm{~F}$ & c. $781 \mathrm{G}>\mathrm{T}$ & p.Gly261Cys & Classical $^{\mathrm{a}}$ \\
\hline & 11.2 & 9.4 & 69 & $\mathrm{~F}$ & c. $621 \mathrm{~T}>\mathrm{A}$ & p.Tyr207* & Classical $^{\mathrm{a}}$ \\
\hline & 9.5 & 9.2 & 51 & $\mathrm{~F}$ & c.584_585insAATAG & p.Arg1961lefs $\star 46$ & Classical \\
\hline & 8.2 & 7.0 & 50 & $\mathrm{~F}$ & c. $187 \mathrm{~T}>\mathrm{C}$ & p.Cys63Arg & Classical \\
\hline & 1.4 & 1.6 & 53 & $\mathrm{~F}$ & c. $644 A>G$ & p.Asn215Ser & Late-onset \\
\hline & 7.6 & 5.1 & 60 & $\mathrm{~F}$ & c. $644 A>G$ & p.Asn215Ser & Late-onset \\
\hline & 3.5 & 3.2 & 60 & $\mathrm{~F}$ & c. $644 A>G$ & p.Asn215Ser & Late-onset \\
\hline & 1.2 & 1.7 & 54 & $\mathrm{~F}$ & c. $644 A>G$ & p.Asn215Ser & Late-onset \\
\hline & 5.8 & 5.4 & 66 & $\mathrm{~F}$ & c. $1079 \mathrm{G}>\mathrm{A}$ & p.Gly360Asp & Classical \\
\hline
\end{tabular}

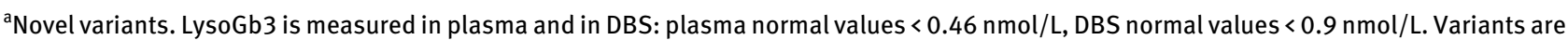
described using the GLA NM_000169.2 transcript reference sequence.

pathogenic and six were novel (Supplementary Table 1). 39 GLA variants are associated with classical Fabry disease and six variants are linked to late-onset Fabry disease. For one variant, the c.728T $>\mathrm{C}$ p.(Leu243Ser), we could not 
assign a clear-cut Fabry phenotype because: (i) the variant is novel; (ii) the patient is female; (iii) hemizygous males have not been detected for this variant. Data of patients harbouring novel GLA variants are summarized in Supplementary Table 2 .

In the analysis on DBS samples, the blood volume that must be considered to obtain a biomarker concentration is strongly related to the Hct value. The Hct measures the volume of red blood cells compared to the total blood volume and is necessary to determine the plasma aliquot in which the biomarker is usually quantified. This parameter strongly influences the volume of blood present in the DBS punch.

When comparing plasma concentration with DBS values, we can use two approaches: we can either use an equation based on Hct levels (known or theoretical) to calculate the corrections due to the change in plasma volume, or we can perform quantitative analysis on the DBS by using a calibration curve prepared with blood with a similar Hct to the blood sample. In the second case, the bias of the sample volume on DBS, due to the presence of red blood cells, is corrected directly by the calibration curve. This second approach was applied in this study: the quantitative analysis on DBS was carried out using calibration curves based on mean age and sex matched hematocrit values.

The correlations of LysoGb3 levels in DBS and plasma are plotted in Figure 2 and show good correlations $\left(R^{2}=0.9717\right)$, indicating that over $97 \%$ of data variability was interpreted by the model, and that the plasma concentration can be confidently estimated by DBS measurements. A few discrepancies were observed in anemic patients, in whom Hct levels differed significantly from the hematocrit of the calibration curve.
A comparison was also performed by Bland-Altman plot, which showed that most of the values obtained were within the confidence interval (95\%) with only three points below the lower limit $(-12.9 \% ; 3 / 104)$, corresponding to the anemic patients (Figure 3). Low hematocrit levels are known to affect quantitative determination on DBS. During DBS sampling, the size spot of a fixed volume of blood depends on blood viscosity; drop with low Hct spread more across the paper. Accordingly, the blood volume contained in a $3.2 \mathrm{~mm}$ punch may be lesser than expected, leading to a significant assay bias [37]. This makes essential the process of accepting samples (pre-analytical phase) when quality of DBS specimens should be evaluated. A pale DBS should make you suspect for a low Hct level and rejected or reported an alert for a possible analytical bias if patient has anemia.

Lyso-Gb3 values were not detected above the assay's LOQ in the healthy population. Thus, the Lyso-Gb3 reference range on DBS was established at $0.9 \mathrm{nmol} / \mathrm{L}$. No agerelated or sex-related changes in LysoGb3 concentrations were observed for healthy subjects.

The proposed method allows small variations in the concentration of LysoGb3 to be observed, even in lateonset patients, who present evident and well integrated signals, but at lower concentrations than patients with classical FD. Mean $( \pm$ SEM) plasma and DBS levels of LysoGb3 in the 30 males with classical FD were $45.6 \pm 9.2$ and $40.6 \pm 9.0 \mathrm{nmol} / \mathrm{L}$, respectively, while in the nine males with late-onset FD, they were $9.2 \pm 3.2$ and $7.9 \pm 2.4 \mathrm{nmol} / \mathrm{L}$, respectively. Mean LysoGb3 levels in 54 females with a GLA variant associated with classical FD were $7.1 \pm 0.6$ (plasma) and $6.4 \pm 0.5 \mathrm{nmol} / \mathrm{L}$ (DBS), while in the nine females with a GLA variant associated with lateonset FD LysoGb3 levels were $1.9 \pm 0.7$ (plasma) and

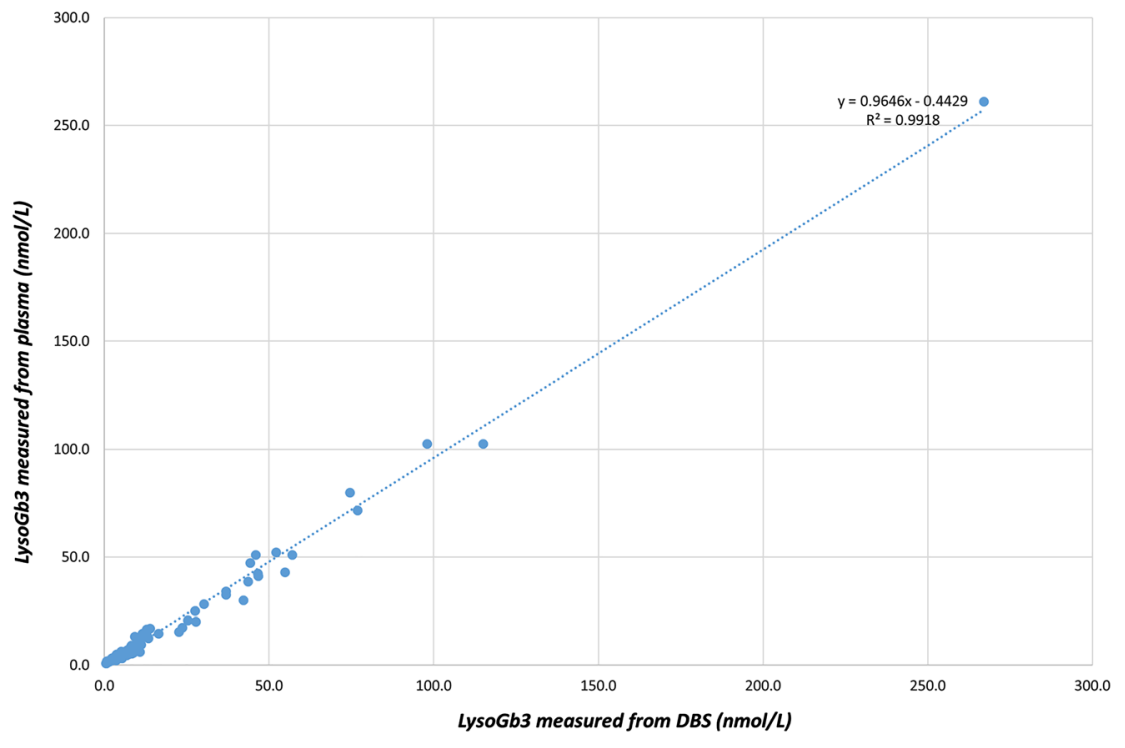

Figure 2: The scatterplot diagrams showing regression analysis of LysoGb3 levels in paired plasma ( $Y$ axis) and DBS ( $X$ axis) specimens from $104 \mathrm{FD}$ patients expressed in $\mathrm{nmol} / \mathrm{L}$. 


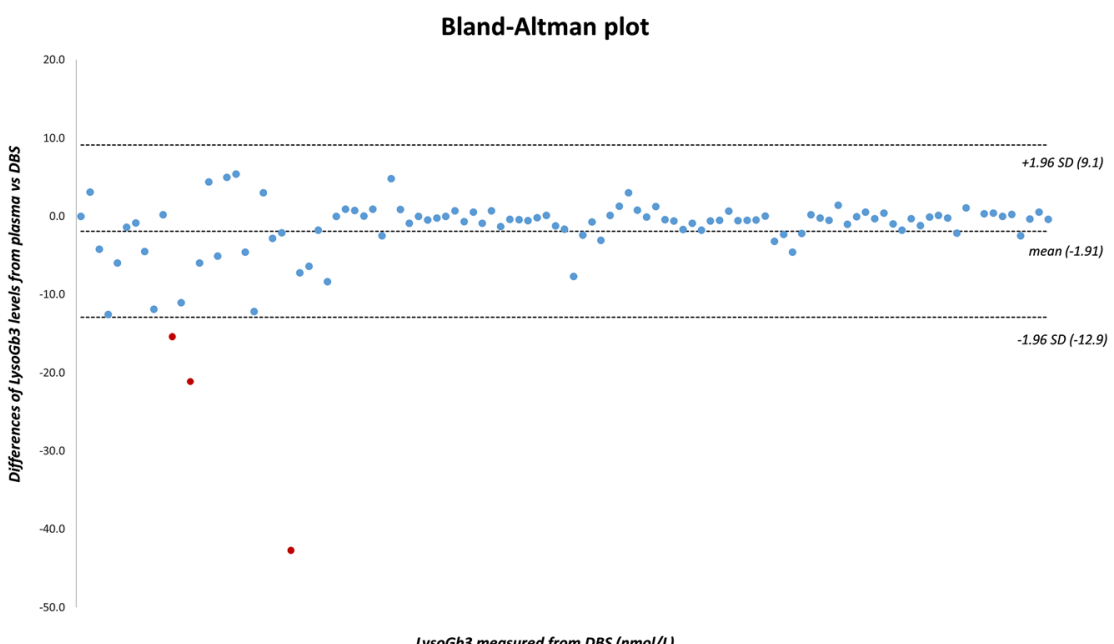

Figure 3: Bland-Altman plot between LysoGb3 value from DBS and plasma in 104 FD subjects.

Mean differences and limits of agreement are represented by dashed lines. The differences lie within the limits of agreement ( \pm 1.96 standard deviations), indicating that the two methods produce comparative results.
A)

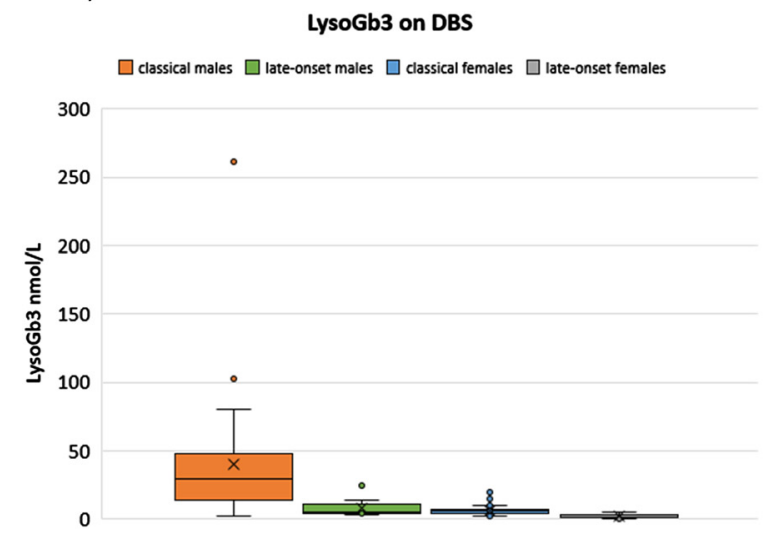

B)

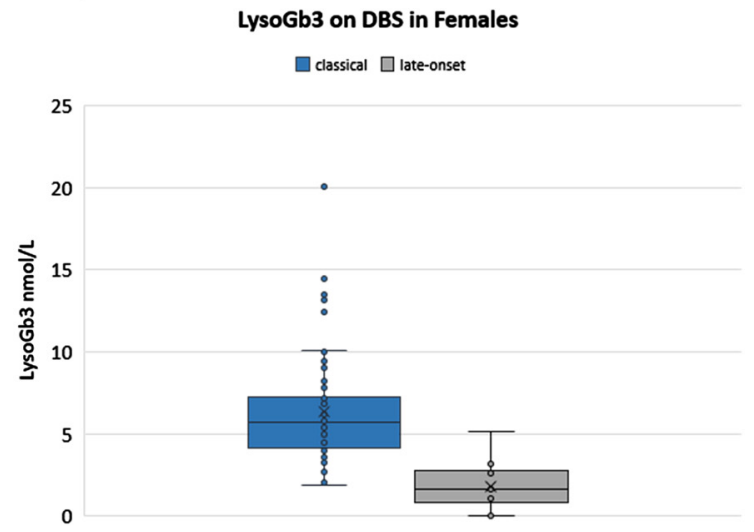

Figure 4: The graphical summary of LysoGb3 measurements on DBS depending on both disease phenotypes and gender. The boxes show the upper (75th) and lower (25th) quartile, the whiskers represent the 5th - 95th percentile. The mean of each dataset is shown as an $\times$. Outliners are plotted as dots. Panel A shows the LysoGb3 distribution in all FD patients tested (males and females). Panel B represents a zoom of the distribution in females

$1.8 \pm 0.5 \mathrm{nmol} / \mathrm{L}$ (DBS). The female having an undetermined phenotype exhibited LysoGb3 levels in plasma and DBS unclear for phenotype assignment, because they could fit with both groups. Figure 4 represents graphically the distribution of LysoGb3 concentrations based on gender and phenotypes; as expected for a linked-disease, there is much more variability in the males' distribution than there is in the females' distribution.

\section{Conclusions}

The advantages of using DBS over plasma have been widely demonstrated in the field of analytical biochemistry. DBS sampling is cheap and easy to collect, transport and store. This work has proved that LysoGb3 assays on DBS are as reliable and accurate as those performed on plasma. This method means that clinicians can easily access LysoGb3 measurement even if the specialized laboratories are far from the clinical center.

Finally, the proposed DBS-based method for the measurement of LysoGb3 may also be applied as a second tier test in positive samples identified though NBS for FD. The combination of a low alpha-Gal A enzyme activity (primary screening result) with a LysoGb3 upper normal limit may reduce the uncertainty in the diagnostic accuracy of FD males associated with the classic phenotype. Unfortunately, there is no statistical difference in LysoGb3 levels at birth in the most of females and late onset males [38]. The adoption of the LysoGb3 second tier test as part of the NBS protocol should be driven by the ethical decision to detect only males susceptible to develop classical FD, preventing identification of FD females and late-onset males. 
Acknowledgments: The authors are grateful to all patients and Anderson-Fabry families who participated in the study Research funding: This research received funding from AIAF: Associazione Italiana Anderson-Fabry onlus.

Author contributions: All authors have accepted responsibility for the entire content of this manuscript and approved its submission.

Competing interests: Authors state no conflict of interest. Informed consent: Informed consent was obtained from all individuals included in this study.

Ethical approval: The local Institutional Review Board of Meyer Hospital of Florence approved this study (No. 116/ 2017).

\section{References}

1. Desnick RJ, loannou YA, Eng CM. $\alpha$-Galactosidase A deficiency: Fabry disease. In: Valle DL, Antonarakis S, Ballabio A, Beaudet AL, Mitchell GA, editors. The online metabolic and molecular bases of inherited disease. New York: McGraw-Hill; 2020.

2. Pinto LL, Vieira TA, Giugliani R, Schwartz IV. Expression of the disease on female carriers of X-linked lysosomal disorders: a brief review. Orphanet J Rare Dis 2010;5:14.

3. Rybová J, Kuchar L, Hulková H, Asfaw B, Dobrovolný R, Sikora J, et al. Specific storage of glycoconjugates with terminal $\alpha$-galactosyl moieties in the exocrine pancreas of Fabry disease patients with blood group B. Glycobiology 2018;28:382-91.

4. Schiffmann R. Fabry disease. Handb Clin Neurol 2015;132: 231-48.

5. Kuipers R, van den Bergh T, Joosten HJ, Le kanne dit Deprez RH, Mannens MM, Schaap PJ. Novel tools for extraction and validation of disease-related mutations applied to Fabry disease. Hum Mutat 2010;31:1026-32.

6. Bokhari SRA, Zulfiqar H, Hariz A. Fabry disease. In: StatPearls. Treasure Island (FL): StatPearls Publishing; 2020.

7. Spada M, Pagliardini S, Yasuda M, Tukel T, Thiagarajan G, Sakuraba $\mathrm{H}$, et al. High incidence of later-onset FABRY disease revealed by newborn screening. Am J Hum Genet 2006;79: 31-40.

8. Hwu WL, Chien YH, Lee NC, Chiang SC, Dobrovolny R, Huang AC, et al. Newborn screening for Fabry disease in Taiwan reveals a high incidence of the later-onset GLA mutation c.936+919G>A (IVS4+919G>A). Hum Mutat 2009;30:1397-405.

9. Ferri L, Malesci D, Fioravanti A, Bagordo G, Filippini A, Ficcadenti $A$, et al. Functional and pharmacological evaluation of novel GLA variants in Fabry disease identifies six (two de novo) causative mutations and two amenable variants to the chaperone DGJ. Clin Chim Acta 2018;481:25-33.

10. Macklin S, Laney D, Lisi E, Atherton A, Smith E. The psychosocial impact of carrying a debated variant in the GLA gene. J Genet Couns 2018;27:217-24.

11. Duro G, Zizzo C, Cammarata G, Burlina A, Burlina A, Polo G, et al. Mutations in the GLA gene and LysoGb3: is it really AndersonFabry disease? Int J Mol Sci 2018;19:3726.
12. Talbot A, Nicholls K. Elevated lyso-Gb3 suggests the R118C GLA mutation is a pathological Fabry variant. JIMD Rep 2019;45: 95-8.

13. Caballero L, Climent V, Hernández-Romero D, Quintanilla MA, de la Morena G, Marín F. Enzyme replacement therapy in Fabry disease: influence on cardiac manifestations. Curr Med Chem 2010;17:1679-89.

14. El Dib R, Gomaa H, Ortiz A, Politei J, Kapoor A, Barreto F. Enzyme replacement therapy for Anderson-Fabry disease: $a$ complementary overview of a Cochrane publication through a linear regression and a pooled analysis of proportions from cohort studies. PLoS One 2017;12:e0173358.

15. Körver S, Vergouwe M, Hollak CEM, van Schaik IN, Langeveld M. Development and clinical consequences of white matter lesions in Fabry disease: a systematic review. Mol Genet Metab 2018;125: 205-16.

16. Ortiz A, Germain DP, Desnick RJ, Politei J, Mauer M, Burlina A, et al. Fabry disease revisited: management and treatment recommendations for adult patients. Mol Genet Metab 2018;123: 416-27.

17. Biferi MG, Cohen-Tannoudji M, García-Silva A, Souto-Rodríguez 0, Viéitez-González I, San-Millán-Tejado B, et al. Systemic treatment of Fabry disease using a novel AAV9 vector expressing $\alpha$-galactosidase A. Mol Ther Methods Clin Dev 2020;20:1-17.

18. Welford RWD, Mühlemann A, Garzotti M, Rickert V, Groenen PMA, Morand 0 , et al. Glucosylceramide synthase inhibition with lucerastat lowers globotriaosylceramide and lysosome staining in cultured fibroblasts from Fabry patients with different mutation types. Hum Mol Genet 2018;27:3392-403.

19. Nowak A, Mechtler TP, Hornemann T, Gawinecka J, Theswet E, Hilz $M J$, et al. Genotype, phenotype and disease severity reflected by serum LysoGb3 levels in patients with Fabry disease. Mol Genet Metab 2018;123:148-53.

20. Nowak A, Beuschlein F, Sivasubramaniam V, Kasper D, Warnock DG. Lyso-Gb3 associates with adverse long-term outcome in patients with Fabry disease. J Med Genet 2021.

21. Togawa T, Kodama T, Suzuki T, Sugawara K, Tsukimura T, Ohashi $\mathrm{T}$, et al. Plasma globotriaosylsphingosine as a biomarker of Fabry disease. Mol Genet Metab 2010;100:257-61.

22. Boutin M, Gagnon R, Lavoie P, Auray-Blais C. LC-MS/MS analysis of plasma lyso-Gb3 in Fabry disease. Clin Chim Acta 2012;414: 273-80.

23. Gold H, Mirzaian M, Dekker N, Joao Ferraz M, Lugtenburg J, Codée JD, et al. Quantification of globotriaosylsphingosine in plasma and urine of fabry patients by stable isotope ultraperformance liquid chromatography-tandem mass spectrometry. Clin Chem 2013;59:547-56.

24. Boutin M, Lavoie P, Abaoui M, Auray-Blais C. Tandem mass spectrometry quantitation of lyso-Gb3 and six related Analogs in plasma for Fabry disease patients. Curr Protoc Hum Genet 2016; 90:17.23.1-9.

25. Polo G, Burlina AP, Kolamunnage TB, Zampieri M, Dionisi-Vici C, Strisciuglio P, et al. Diagnosis of sphingolipidoses: a new simultaneous measurement of lysosphingolipids by LC-MS/MS. Clin Chem Lab Med 2017;55:403-14.

26. Beasley J, McCaw P, Zhang H, Young SP, Stiles AR. Combined analysis of plasma or serum glucosylsphingosine and globotriaosylsphingosine by UPLC-MS/MS. Clin Chim Acta 2020; 511:132-7. 
27. McClendon-Weary B, Putnick DL, Robinson S, Yeung E. Little to give, much to Gain-what can you do with a dried blood spot? Curr Environ Health Rep 2020;7:211-21.

28. Malsagova K, Kopylov A, Stepanov A, Butkova T, Izotov A, Kaysheva A. Dried blood spot in laboratory: directions and prospects. Diagnostics (Basel) 2020;10:248.

29. Olivera S, Iñiguez C, García-Fernández L, Sierra JL, Camón AM, Menao S, et al. Usefulness of lyso-globotriaosylsphingosine in dried blood spots in the differential diagnosis between multiple sclerosis and Anderson-Fabry's disease. Mult Scler Relat Disord 2020;38:101466.

30. Gatterer C, Gaggl M, Mundigler G, Rommer P, Graf S, SunderPlassmann G. Agreement of dried blood spot lyso-Gb3 concentrations obtained from different laboratories in patients with Fabry disease. Clin Chem Lab Med 2020;58:e275-8.

31. Delarosa-Rodríguez R, Santotoribio JD, Paula HA, González-Meneses A, García-Morillo S, Jiménez-Arriscado P, et al. Accuracy diagnosis improvement of Fabry disease from dried blood spots: enzyme activity, lyso-Gb3 accumulation and GLA gene sequencing. Clin Genet 2021.

32. Johnson B, Mascher H, Mascher D, Legnini E, Hung CY, Dajnoki A, et al. Analysis of lyso-globotriaosylsphingosine in dried blood spots. Ann Lab Med 2013;33:274-8.

33. Nowak A, Mechtler T, Kasper DC, Desnick RJ. Correlation of LysoGb3 levels in dried blood spots and sera from patients with classic and Later-Onset Fabry disease. Mol Genet Metab 2017; 121:320-4.

34. Polo G, Burlina AP, Ranieri E, Colucci F, Rubert L, Pascarella A, et al. Plasma and dried blood spot lysosphingolipids for the diagnosis of different sphingolipidoses: a comparative study. Clin Chem Lab Med 2019;57:1863-74.

35. Biegstraaten M, Arngrímsson R, Barbey F, Boks L, Cecchi F, Deegan PB, et al. Recommendations for initiation and cessation of enzyme replacement therapy in patients with Fabry disease: the European Fabry Working Group consensus document. Orphanet J Rare Dis 2015;10:36.

36. ICH Harmonised Guideline. Bioanalytical method validation M10. Available from: https://www.ema.europa.eu/en/documents/ scientific-guideline/draft-ich-guideline-m10-bioanalyticalmethodvalidation-step-2b_en.pdf [Accessed 21 Aug 2020].

37. Denniff $P$, Spooner $N$. The effect of hematocrit on assay bias when using DBS samples for the quantitative bioanalysis of drugs. Bioanalysis 2010;2:1385-95.

38. Malvagia S, Forni G, Ombrone D, la Marca G. Development of strategies to decrease false positive results in newborn screening. Int J Neonatal Screen 2020;6:84.

Supplementary Material: The online version of this article offers supplementary material (https://doi.org/10.1515/cclm-2021-0316). 